\title{
Development of melanopsin-based irradiance detecting circuitry
}

\author{
David S McNeill ${ }^{1 \dagger}$, Catherine J Sheely ${ }^{1 \dagger}$, Jennifer L Ecker ${ }^{1}$, Tudor C Badea, ${ }^{3}$, Duncan Morhardt ${ }^{2}$, William Guido ${ }^{2 *}$, \\ Samer Hattar ${ }^{1 *}$
}

\begin{abstract}
Background: Most retinal ganglion cells (RGCs) convey contrast and motion information to visual brain centers. Approximately $2 \%$ of RGCs are intrinsically photosensitive (ipRGCs), express melanopsin and are necessary for light to modulate specific physiological processes in mice. The ipRGCs directly target the suprachiasmatic nucleus (SCN) to photoentrain circadian rhythms, and the olivary pretectal nucleus (OPN) to mediate the pupillary light response. How and when this ipRGC circuitry develops is unknown.
\end{abstract}

Results: Here, we show that some ipRGCs follow a delayed developmental time course relative to other imageforming RGCs. Specifically, ipRGC neurogenesis extends beyond that of other RGCs, and ipRGCs begin innervating the SCN at postnatal ages, unlike most RGCs, which innervate their image-forming targets embryonically. Moreover, the appearance of ipRGC axons in the OPN coincides precisely with the onset of the pupillary light response.

Conclusions: Some ipRGCs differ not only functionally but also developmentally from RGCs that mediate patternforming vision.

\section{Background}

The mammalian retina detects, processes, and signals light information to the brain to form images and modulate physiological processes. As the sole output neurons of the retina, most retinal ganglion cells (RGCs) encode visual information such as motion and contrast, whereas $2 \%$ of RGCs contain the photopigment melanopsin and signal irradiance information to the brain to control circadian rhythms, sleep, and the pupillary light response (PLR) [1-4]. These intrinsically photosensitive RGCs (ipRGCs) project directly to brain regions that mediate light-dependent physiological processes, notably the suprachiasmatic nucleus (SCN), which is the master circadian pacemaker, and the olivary pretectal nucleus (OPN), which controls PLR $[5,6]$. Genetic ablation of ipRGCs leaves image formation intact, but severely impairs the effects of light on circadian rhythms, sleep, and PLR $[3,4]$. Thus, the pathways for light input to

\footnotetext{
* Correspondence: wguido@vcu.edu; shattar@jhu.edu

+ Contributed equally

'Department of Biology, Johns Hopkins University, Baltimore, MD 21218, USA ${ }^{2}$ Department of Anatomy and Neurobiology, Virginia Commonwealth

University, Richmond, VA 23298, USA

Full list of author information is available at the end of the article
}

image-formation and regulation of physiological processes diverge at the level of the RGCs.

Until recently, the majority of research on ipRGCs has focused on the initially identified subtype (M1), which arborizes in the OFF sublamina of the inner plexiform layer and labels with expression of the tauLacZ reporter from the endogenous melanopsin locus $\left(O p n 4^{\text {taulac }}\right)$. However, a more sensitive reporter system utilizing expression of cre recombinase from the endogenous melanopsin locus $\left(\mathrm{Opn} 4^{\text {cre }}\right)$ revealed additional subtypes of ipRGCs beyond the previously identified M1 subtype. Some of these newly identified subtypes target the dorsal lateral geniculate nucleus (dLGN), a thalamic relay center for visual processing. This work also demonstrated that ipRGCs are capable of supporting visual processing, indicating that some ipRGCs have overlapping targeting and function with other RGCs [7].

How ipRGCs develop in order to fulfill their unique physiological functions remains unclear. To begin answering this question, we examined the timing of ipRGC neurogenesis, axonal targeting, and the developmental onset of pupil constriction, an ipRGC-mediated functional output.
Ciomed Central

C 2011 McNeill et al; licensee BioMed Central Ltd. This is an Open Access article distributed under the terms of the Creative Commons Attribution License (http://creativecommons.org/licenses/by/2.0), which permits unrestricted use, distribution, and reproduction in any medium, provided the original work is properly cited. 


\section{Results}

Birth of ipRGCs continues beyond that of other RGCs

To compare ipRGC neurogenesis with other RGCs, we labeled terminally dividing cells with 5-ethynyl-2'deoxyuridine (EdU) on individual days of embryonic development (Figure 1A,B). We used immunostaining for the transcription factor Brn3a to label a subset of RGCs separate from ipRGCs [8] and the melanopsin-tau-LacZ $\left(\mathrm{Opn} 4^{\text {tauLacZ/+}}\right)$ reporter to detect ipRGCs. The tau-lacZ reporter expresses tau- $\beta$-galactosidase, which is cytoplasmic and is predominantly trafficked to axons. The Opn $4^{\text {tauLacZ/+ }}$ reporter primarily labels the M1 subtype in adult mice [9], but it remains unclear if this holds true during development since ipRGC subtypes cannot be anatomically identified prior to dendritic arborization. Tissue was analyzed at postnatal day 0 (P0), which is a time point after RGC birth but before the major wave of RGC death $[10,11]$. The onset of ipRGC neurogenesis is consistent with that observed for Brn3a-positive RGCs as well as the RGC population as a whole, which begins at embryonic day 11 (E11) [11,12]. EdU injections on subsequent days during development revealed that the majority of ipRGCs are born from E11 to E14 (Figure 1C; Additional file 1). Although the Brn3a population shows a sharp decline in birth after E15, with only one positive cell observed at E16, a significant proportion of ipRGCs are born through E18 (Figure 1B,C).

To determine when melanopsin is first expressed in the developing retina, we used three different labeling methods: a melanopsin antibody and two genetic reporter mouse lines, Opn $4^{\text {tauLacZ/+ }}$ [5] and Opn $4^{\mathrm{Cre} /+}$ in conjunction with the $Z / A P$ reporter, which does not depend on transcription of the melanopsin locus for signal strength $[7,13]$. All three labeling techniques indicated that melanopsin is expressed from E15 onwards since there was a complete absence of staining at E14 (Figure 1D-F). Melanopsin expression is first detected at a similar time point in rats [14]. While it is possible that melanopsin is expressed at low levels prior to E15, it is unlikely because the genetic reporters used in this study have high sensitivity due to enzymatic signal amplification. At E15 some melanopsin-positive cells were detected in the ganglion cell layer, while others appeared to be migrating from the neuroblast layer to the ganglion cell layer (Figure 1D-F, arrows). Although melanopsin positive cells were absent from the peripheral retina at E15, during subsequent days of development the region of labeled cells subsequently expanded (Figure $1 \mathrm{G}$, arrows), reaching the ciliary margin by P0.

ipRGCs innervate their main target, the suprachiasmatic nucleus, postnatally

To determine when RGC axonal projections from the eye reach the $\mathrm{SCN}$, we used four different labeling methods. Total RGC axonal projections were labeled with cholera toxin B subunit (CTB), which revealed that RGC axons begin to innervate the contralateral borders of the SCN soon after birth (Figure 2A), similar to rats $[15,16]$. At P3 to P4 axons from both eyes emerged at the midline to provide bilateral innervation of the SCN beginning caudally and achieving an adult-like pattern by the second postnatal week. To specifically label ipRGC axons, we used the Opn $4^{\text {tauLacZ/+ }}$ mice, which directly report transcription from the melanopsin locus and the $O p n 4^{\mathrm{Cre} /+} ; \mathrm{Z} / A P$ reporter, which does not depend on the melanopsin locus for signal strength. The innervation patterns revealed by all three labeling methods are identical (Figure 2A-C) and directly correspond to the spatial and temporal progression of cFos induction in the SCN by light in early postnatal mice [17]. Even though the SCN is innervated postnatally (Figure $2 \mathrm{~A}-\mathrm{C})$, ipRGC axons are present in the chiasm on the ventral surface of the SCN as early as E17 (Figure 3). In contrast, axons from cholera toxin labeled RGCs have already entered more caudal targets, such as the lateral geniculate nucleus and the pretectum by P1 (Figures 2D and 4A) [18]. To directly compare the innervation patterns of ipRGCs to a larger population of RGCs, we used an alkaline phosphatase reporter that labels RGCs positive for the transcription factor Brn3b (Figure 2E) [18]. We confirmed that the $\mathrm{SCN}$ is innervated later than visual targets such as the LGN, where fibers enter by $\mathrm{P} 1$, and the pretectum, where fibers enter by E17 (Figure 2F, arrows). Serial sections at different developmental time points reveal the complete spatial and temporal progression of $O p n 4^{\text {tauLacZ/+ }}$-labeled axons in the SCN (Figure 3).

\section{Onset of the pupillary light response corresponds to emergence of ipRGC axons in the OPN shell}

Next we used CTB, Opn $4^{\text {tauLacZ/+ }}$, and $\mathrm{Opn} 4^{\mathrm{Cre} /+} ; \mathrm{Z} / \mathrm{AP}$ labeling techniques to determine the development of retinal projections to the OPN, which mediates the PLR [19]. Both CTB labeling of RGC axons and $\mathrm{Opn} 4^{\mathrm{Cre} /{ }^{+}} ; \mathrm{Z} /$ $A P$ labeling of ipRGC axons revealed innervation of the OPN from birth, achieving an adult-like morphology by P7 (Figure 4A,B). In contrast to this early postnatal labeling throughout the OPN (Figure 4A,B), the Opn4tauLacZ/+-labeled ipRGC axons showed only faint labeling in the shell of the OPN starting at P7 and reached adult morphology at P14 (Figure 4C). Similarly, Opn4 ${ }^{\mathrm{Cre} /{ }^{+}} ; \mathrm{Z} /$ $A P$-labeled ipRGC axons are present throughout the LGN from birth, including the dorsal LGN, which is an important relay for visual information. In contrast, the Opn $4^{\text {tauLacZ/+}}$-labeled ipRGC axons do not begin to appear in the intergeniculate leaflet of the LGN until about P3 (Figure 5). Thus, for both the OPN and the LGN, the Opn $4^{\text {tauLacZ/+ }}$-labeled ipRGC axons appear to 


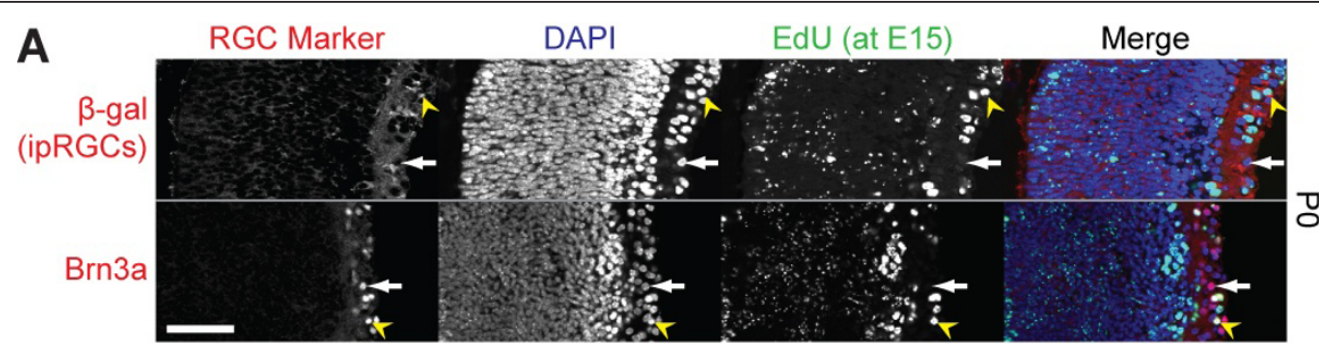

B
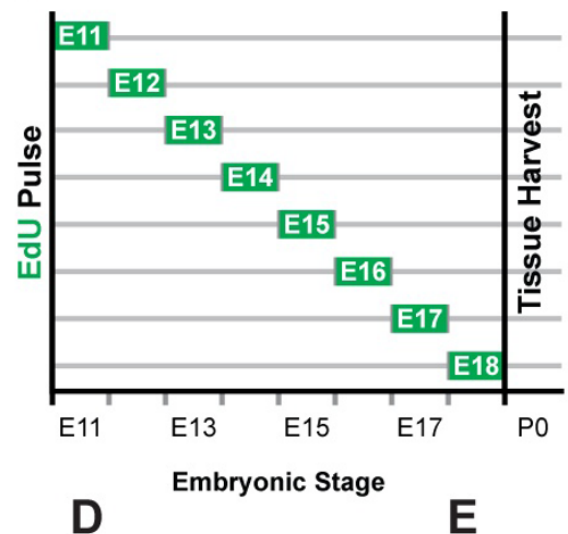

Anti-melanopsin DAPI

\section{E}

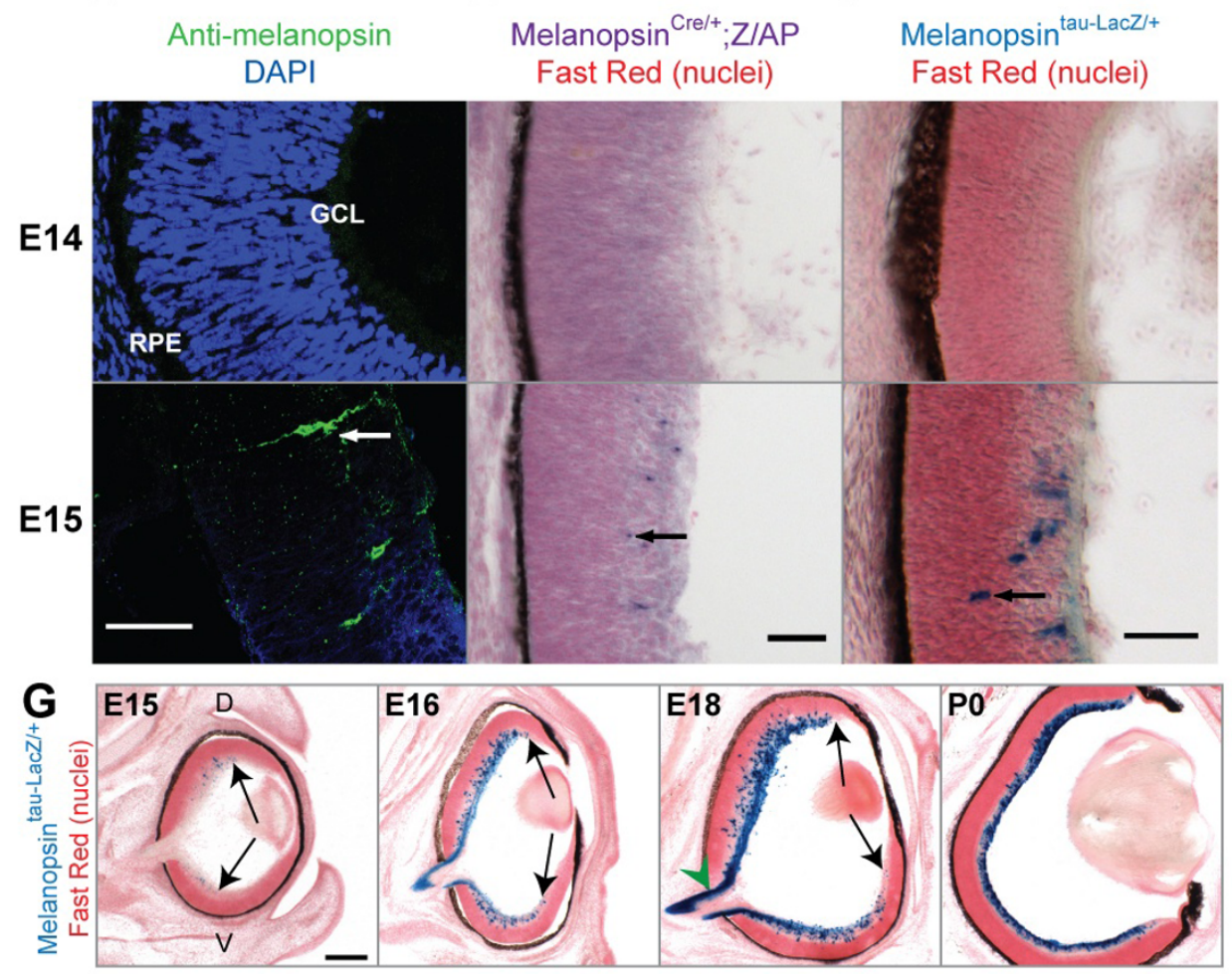

Figure 1 Birth and melanopsin expression of ipRGCs. (A,B) The birthdates of ipRGCs were compared to Brn3a-positive RGCs (representative images in (A), paradigm in (B)). (C) For each time point, we determined the proportion of ipRGCs labeled with EdU; asterisks indicate significant difference between ipRGCs and Brn3a ( $t$-test, $P<0.05$ ). Representative sections with double-labeled RGCs (yellow arrowheads) and EdU-negative RGCs (white arrows) from postnatal day 0 (P0) retinas pulsed with EdU at embryonic day 15 (E15) are shown in (A). Note that the ipRGC marker beta-galactosidase ( $\beta$-gal), is cytoplasmic, while Brn3a and $E d U$ are nuclear. For all EdU time points, $n=3$ to 4 retinas per time point, mean \pm standard error of the mean. (D-F) Melanopsin (Opn4) expression begins at E15 based on immunofluorescence (D), and the Melanopsin ${ }^{\mathrm{Cr} /+} ; \mathrm{Z} / \mathrm{AP}$ (E) and MelanopsintaulacZ/t genetic labeling methods (F). The Melanopsin ${ }^{\text {Cre/ }} ; Z$ Z/AP labels all ipRGC subtypes and does not depend on the melanopsin locus for signal strength. Arrows in D-F denote migrating cells. GCL; Ganglion Cell Layer and RPE; Retinal Pigmented Epithelium. (G) Coronal sections from Melanopsin ${ }^{\text {tauLacZ/+ }}$ mice show an initial lack of ipRGCs in the periphery at E15, which is entirely filled in by P0 (arrows; D, dorsal; $V$, ventral). Note the lack of X-gal staining within the central optic nerve at E18 (G, green arrowhead). Scale bars: $50 \mu \mathrm{m}$ (A,D-F); $100 \mu \mathrm{m}$ (G). 


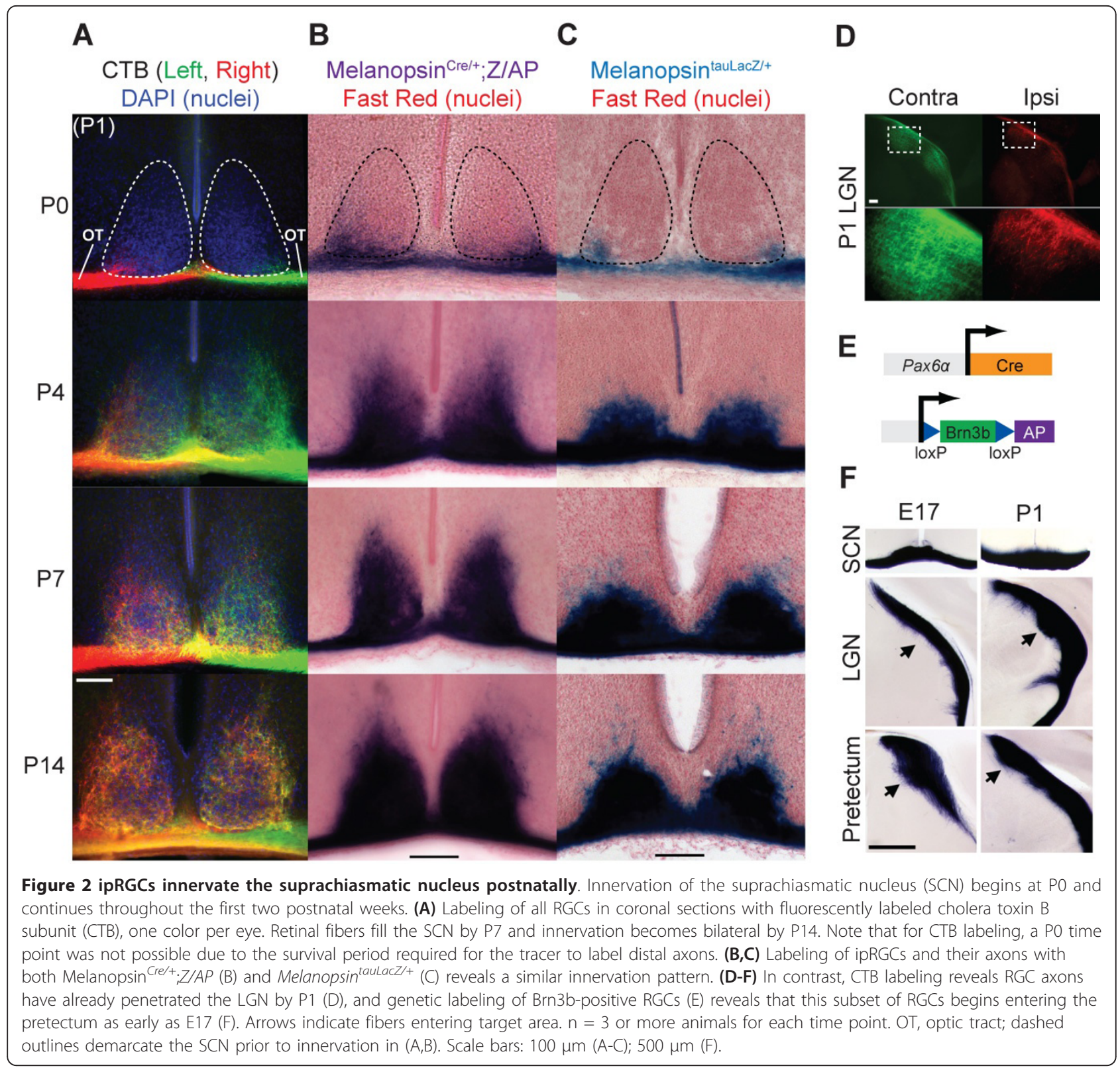

innervate these regions later than the other ipRGC axons that label with the $\mathrm{Opn} 4^{\mathrm{Cre} /+} ; \mathrm{Z} / \mathrm{AP}$ reporter.

Next we determined if the appearance of ipRGC axons in the OPN correlates with the onset of the functional output of the OPN, the PLR. Under high light intensity $\left(1.35 \times 10^{16}\right.$ photons $\left./ \mathrm{cm}^{2} \mathrm{~s}\right)$, we observed rudimentary PLR starting at P7 and substantial responses from P10 onwards (representative images shown in Figure 4E, data summarized in Figure 4F). This onset of the PLR is similar to that in rats [20]. The lack of PLR prior to P7 was not limited by development of the ciliary muscle since from P6 to P8 the cholinergic agonist carbachol constricted the pupil significantly more than light (oneway ANOVA, $P<0.0001$; Tukey post hoc, $P<0.001$ ).
From P10 onwards, the light intensity we used constricted the pupil to levels similar to carbachol, indicating that it is bright enough to cause maximal constriction (one-way ANOVA, Tukey post hoc, $P>$ 0.05 for P10 to P14). Interestingly, we noticed a baseline constriction of the pupil in the dark before light exposure from P9 to P14, perhaps caused by holding the lid margins open (see Materials and methods and paradigm in Figure 4D). This constriction was always significantly less than the constriction due to light (one-way ANOVA, $P<0.0001$; Tukey post hoc, $P<0.01$ for $\mathrm{P7}$ and $P<0.001$ for P8 to P14). Application of the topical anesthetic proparacaine did not have a significant effect on this baseline constriction; thus, it was unlikely to be 


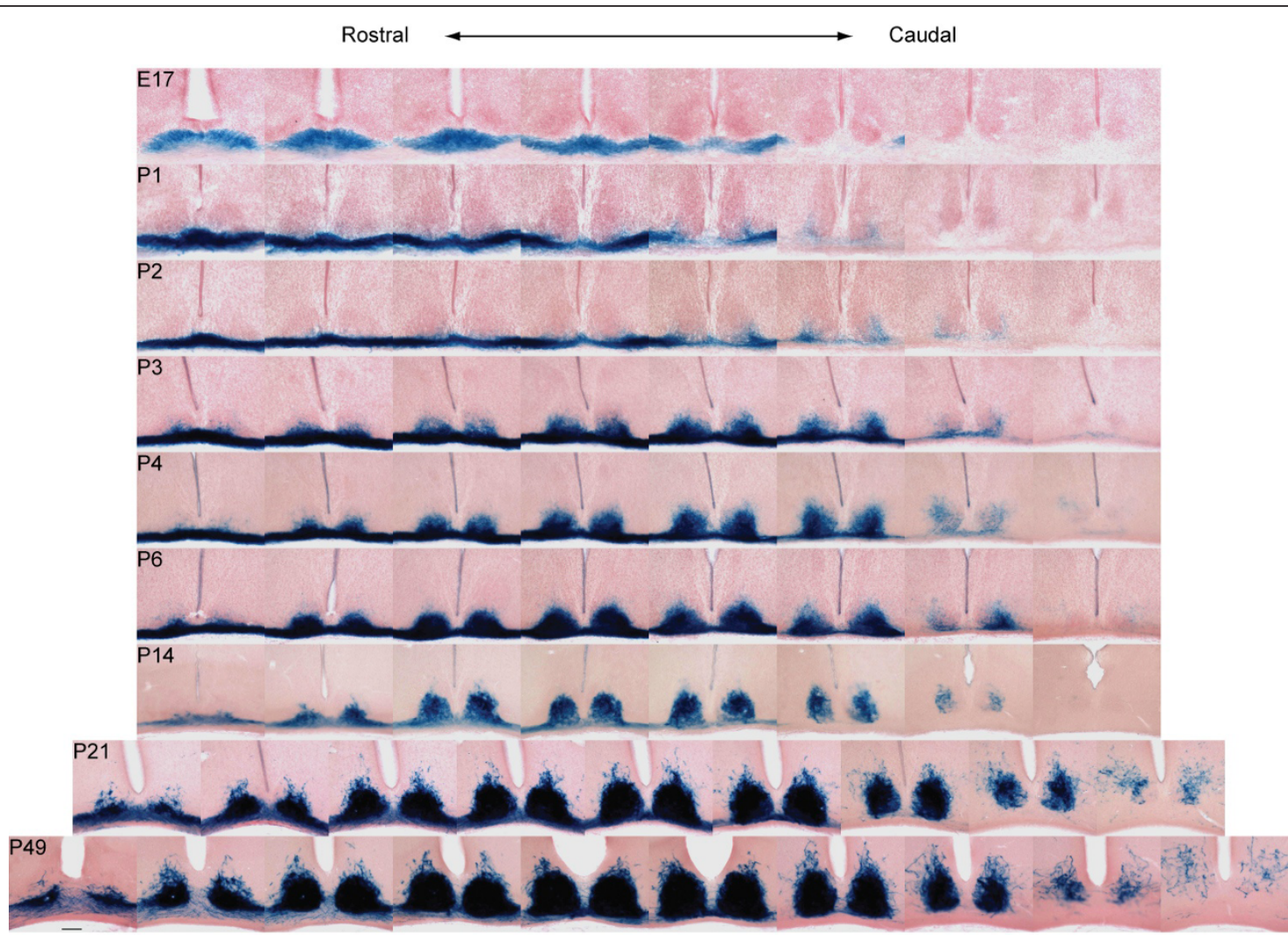

Scale bar $100 \mu \mathrm{m}$

Figure 3 Spatial and temporal progression of SCN innervation. Serial 50- $\mu \mathrm{m}$ coronal sections from individual brains of Melanopsin ${ }^{\text {taulacz/ }+}$ mice at different time points reveal the spatial and temporal progression of ipRGC innervation of the SCN. Nuclei are counterstained with Vector Nuclear Fast Red. Scale bar: $100 \mu \mathrm{m}$.

caused by the direct sensation of the forceps (data not shown). In conclusion, light consistently induced pupil constriction starting at P7, coincident with the appearance of ipRGC axons in the shell of the OPN, a crucial connection in the PLR circuit $[4,9]$.

\section{Discussion}

Over a century of research has focused on the development of RGCs and their role in image formation. Work over the last decade has revealed an intrinsically photosensitive subset of RGCs (ipRGCs) that signal irradiance information to brain regions that modulate processes such as sleep, circadian rhythms, and PLR $[3,4]$. Here, we provide the first direct examination of ipRGC birth and axonal targeting in the brain. Our data reveal a broad diversity of developmental parameters within the ipRGC population, with the development of some ipRGCs diverging from the majority of other RGCs.

\section{A subset of ipRGCs is born later than other RGCs}

Previous studies have shown that RGCs are born between E11 and E18 [12,21]. We find that most ipRGCs are born between E11 and E15, similar to other RGCs [11,12,21]. In stark contrast to the Brn3a-positive RGCs, we observe a significant amount of ipRGC neurogenesis after E15 (Figure 1C). Interestingly, Math5, a transcription factor involved in RGC fate determination, is downregulated at E16 [22]. Thus, this late born ipRGC cohort may be specified independent of Math5. In agreement with this idea, some ipRGCs remain in the Math5 knockout [23]. The early born ipRGCs may share an overlapping function with other RGCs, such as the ipRGCs that are capable of supporting pattern vision [7], and the later born ipRGCs may preferentially target non-image forming areas that are innervated later, such as the SCN and the shell of the OPN. Such a divergence could be determined by the identification of molecular markers for individual ipRGC subtypes. 


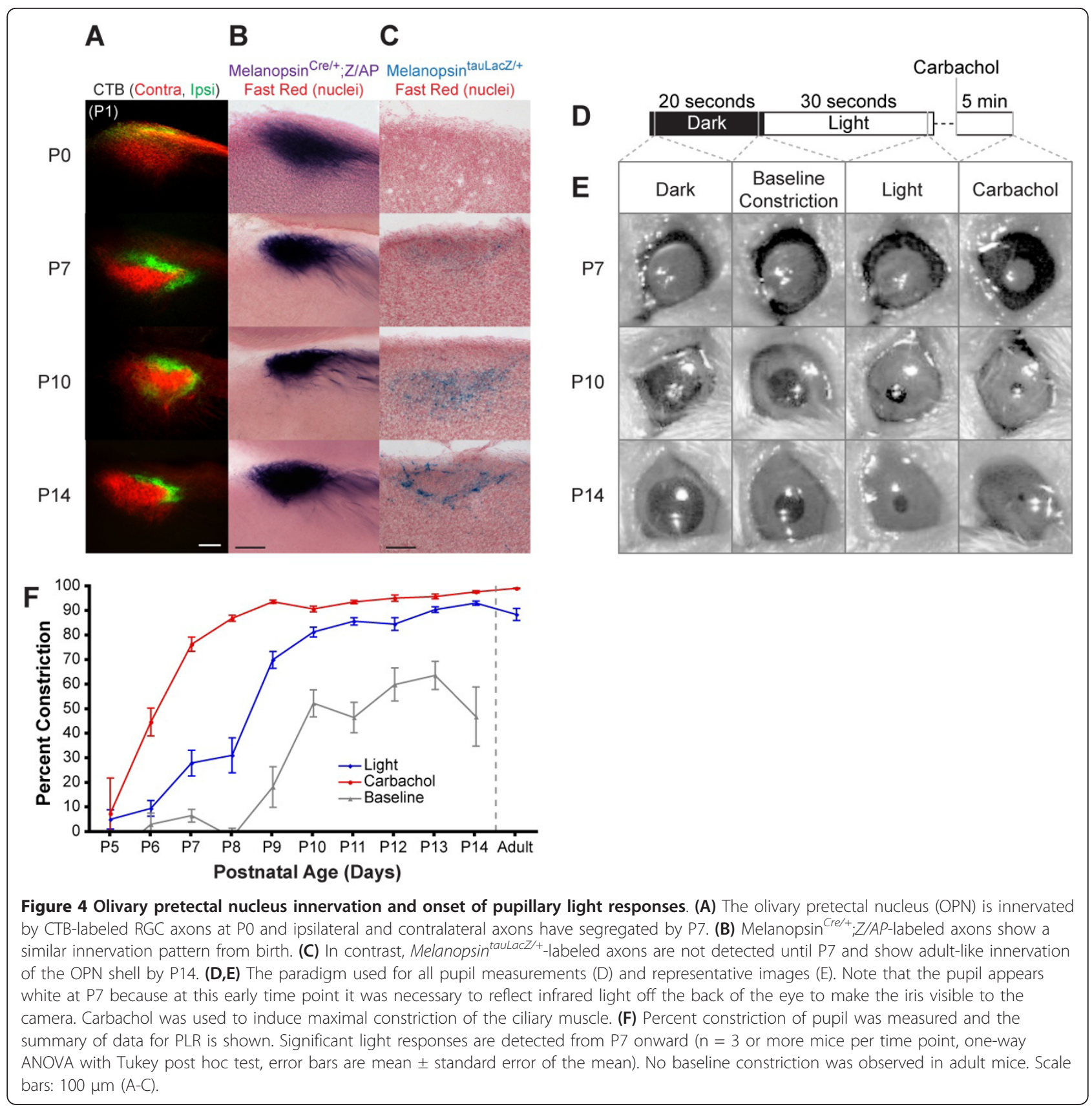

\section{The $\mathrm{SCN}$ is innervated later than image-forming retinal targets}

During embryonic development, the majority of RGC axons project through the optic chiasm past the overlying SCN to target visual brain centers such as the LGN and the superior colliculus (Figure 2F) $[15,16,24,25]$. In contrast, cholera toxin labeling of RGC fibers showed that retinal axons enter the SCN postnatally. The lateral edges of the SCN fill first according to CTB labeling and both the Opn $4^{\text {tauLacZ/+ }}$ and $O p n 4^{\mathrm{Cre} /+} ; \mathrm{Z} / \mathrm{AP}$ reporters, which in turn closely match a previous report that exposure to bright light can induce cFos expression in the lateral edges of the SCN in newborn mice [17]. The spatio-temporal agreement of ipRGC axonal labeling with the induction of cFos expression by light supports the well-established idea that most of the RGCs that innervate the SCN express melanopsin [4-6,9,26].

There are at least three possible explanations for this later innervation of the SCN by ipRGCs. First, ipRGC axons may follow a later time course compared to other RGCs. Such a delay in targeting would correspond with the later born ipRGCs we observe. Second, ipRGC axons that 


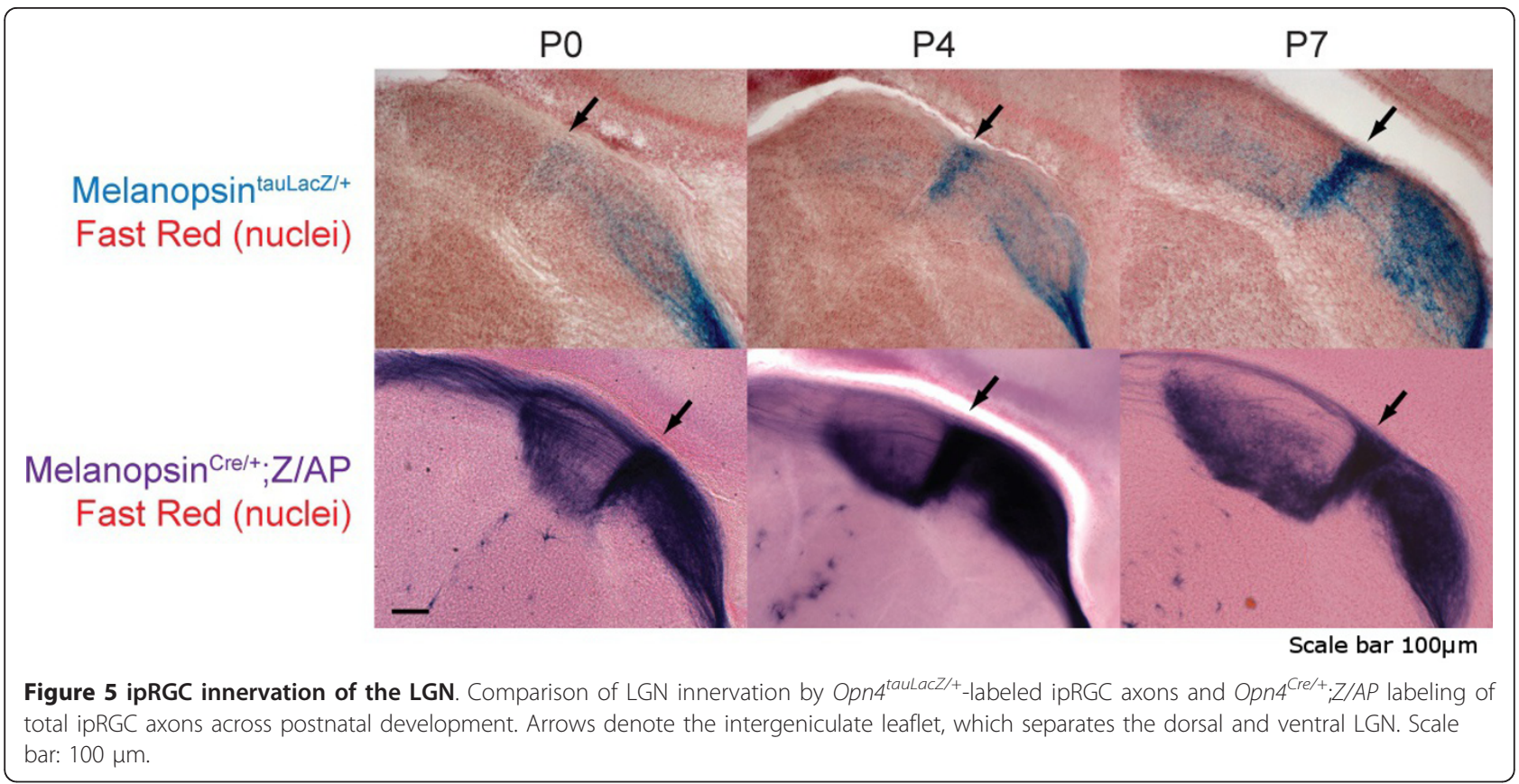

innervate the SCN may reach the chiasm earlier in development, but stall there until P0. Though we do observe ipRGC axons in the chiasm ventral to the SCN from E17, it remains to be determined if these specific ipRGC axons subsequently innervate the SCN (Figure 3). It is doubtful that innervation of the $\mathrm{SCN}$ is dependent upon functional maturation of target cells since the SCN begins oscillating before birth $[27,28]$. Finally, the SCN could be innervated by collaterals from RGC axons that have already passed through the chiasm to more distal targets in the LGN, pretectum or colliculus $[29,30]$. Indeed, such a delay in collateralization of RGC axons into the SCN has previously been reported [15]. Since most RGC axons pass by the $\mathrm{SCN}$ to enter visual targets embryonically (Figure $2 \mathrm{~F}$ ), this temporal separation could be a means for preventing nonipRGC axons from aberrantly terminating in the SCN. If this is the case, the ipRGCs that innervate the SCN may be uniquely receptive to a yet undetermined signal from the $\mathrm{SCN}$. Expression of such factors may complement the spatial and temporal characteristics of ipRGC innervation of the SCN during development (Figure 3).

\section{Onset of PLR correlates with emergence of ipRGC axons in the OPN shell}

The PLR is mediated by retinal input to the OPN, which can be divided into core and shell regions. The shell is defined by parvalbumin and calbindin-D expression [31,32], and is innervated by the M1 subset of ipRGCs, which label with Opn4 $4^{\text {tauLacZ/+ }}[6,9]$. This connection of M1 ipRGCs to the OPN shell is crucial to the PLR circuit, since genetic ablation of the Opn $4^{\text {tauLacZ/++}}$-labeled ipRGCs results in severe impairment of the pupillary light response [4].
In agreement with these data, the Edinger-Westphal nucleus, which is the brain relay downstream of the OPN in the PLR circuit, is primarily innervated by axons from neurons in the shell of the OPN [9].

Our data suggest that two subtypes of ipRGCs innervate the OPN with different spatial and temporal profiles. CTB labeling of RGC axons and Opn $4^{\mathrm{Cre} /+} ; \mathrm{Z} / \mathrm{AP}$ labeling of all ipRGCs reveal robust innervation of the OPN from birth. In contrast, the $\mathrm{Opn} 4^{\mathrm{tauLacZ} /+}$ reporter only shows labeling of ipRGC axons in the OPN starting in the second postnatal week. This delayed labeling is limited to the shell region of the OPN, similar to adult labeling with the Opn $4^{\text {tauLacZ/+ }}$ reporter $[5,6]$. Thus, it appears that the M1 ipRGCs innervate the OPN shell later than the ipRGCs that innervate the core. We observe a similar temporal separation between $\mathrm{Opn} 4^{\mathrm{Cre} /{ }^{+}} ; \mathrm{Z} / \mathrm{AP}$-labeled ipRGCs that innervate the LGN and the Opn $4^{\text {tauLacZI }}$ ${ }^{+}$-labeled subset that innervate the intergeniculate leaflet (Figure 5). Thus, the Opn $4^{\mathrm{Cre} /+} ; \mathrm{Z} / \mathrm{AP}$ reveals that the ipRGCs that innervate classical visual targets such as the dLGN follow a developmental paradigm similar to other RGCs, while the Opn $4^{\text {tauLacZ/+ }}$ labeled ipRGCs appear to innervate their specific targets later.

To explore the functional output of the differing ipRGC projections to the OPN, we measured the PLR of postnatal mice. We first detected a rudimentary PLR at P7, coincident with the appearance of the Opn $4^{\text {tauLacZ/+ }}$-labeled axons in the OPN shell. Although we cannot rule out the role that other relay centers play in determining the onset of PLR, it is striking that the onset of PLR coincides with the appearance of Opn $4^{\text {tauLacZ/+ }}$-labeled axons in the OPN, a crucial connection for the PLR. 
This early PLR must be driven by melanopsin since it accounts for all retinal photoreception through at least P10 [17,33,34] and synaptic connections between retinal layers are not functional until eye opening at P12 to P14 [35]. While it remains unclear why PLR begins several days prior to eye opening, it agrees with mounting evidence that ipRGCs comprise the first functioning photoreceptive system during development $[17,34]$.

\section{Conclusions}

Together, these data indicate diversity in the developmental trajectory of ipRGCs and form a foundation to explore the molecular mechanisms that govern the specification and wiring of this distinct visual subsystem.

\section{Materials and methods}

Animals

All procedures were performed on mice bred on a mixed C57BL/6x129 background in accordance with the IACUC protocols of Johns Hopkins University and Virginia Commonwealth University Medical Center. All mice were housed in a 12:12 hour light:dark cycle.

\section{X-gal staining}

Tissue from Opn $4^{\text {tauLacZ/+ }}$ mice was prepared as previously described [6], stained for 2 days in X-gal and counterstained with Vector Nuclear Fast Red.

\section{Alkaline phosphatase labeling}

We used previously generated mice that express Cre recombinase from the endogenous melanopsin locus [7]. The experimental animals used in this study were obtained by mating Opn $4^{\mathrm{Cre} / \mathrm{Cre}}$ mice to $\mathrm{Z} / \mathrm{AP}$ reporter mice [13].

\section{Eye injections}

To visualize retinofugal projections originating from each eye, intravitreal injections of the fluorescently conjugated anterograde tracer CTB were performed as described in [36]. Animals were given an 18- to 36-hour survival period.

\section{Melanopsin immunolabeling}

Tissue was cryosectioned at $16 \mu \mathrm{m}$ in the coronal plane and stained with rabbit $\alpha$-melanopsin (1:2,000; a gift from I Provencio) and incubated on slides for 4 days at $4{ }^{\circ} \mathrm{C}$. Slides were incubated with donkey-anti-rabbit Alexa 488 (1:500; Molecular Probes; Carlsbad, CA USA). Slides were mounted in AntiFade (Molecular Probes) with DAPI.

\section{Birthdating}

Females pregnant with $O p n 4^{\text {tauLacZ/+ }}$ pups were injected with EdU (Invitrogen; Carlsbad, CA USA) every 3 hours over a 24-hour period [12]. Tissue was collected at P0, sectioned at $18 \mu \mathrm{m}$ in the coronal plane, and stained with chicken anti- $\beta$-galactosidase (1:600; Millipore; Billerica, MA USA) or mouse anti-Brn3a (1:25; Millipore) incubated overnight at $4{ }^{\circ} \mathrm{C}$ followed by donkey antichicken Alexa 546 (1:500; Molecular Probes) or goat anti-mouse Alexa 546 (1:500; Molecular Probes). Sections were imaged at $40 \times$ with a Zeiss LSM 510 META confocal microcsope. The proportion of ipRGCs or Brn3a-positive cells born on a specific day was determined for samples of more than 100 cells per retina.

\section{Pupillary light response}

PLR was evoked using a 473-nm light-emitting diode at $1.35 \times 10^{16}$ photons $/ \mathrm{cm}^{2} \cdot \mathrm{s}$, [37]. To view the pupil before natural eye opening (P12 to P14), it was necessary to separate the eyelids along the line of fusion and gently hold them open with curved forceps. All animals were dark-adapted for at least 1 hour before measurements, which were restricted to the middle of the light portion of the day. One eye of each mouse was monitored under infrared light with a Sony DCR-HC96 video camera. The percentage of pupil constriction was calculated by comparing the pupil area at the end of each treatment to the pupil area in the dark at the beginning of the recording. To measure any baseline constriction due to handling or other non-light stimuli, percent constriction was measured after holding the mouse in the dark for 20 seconds. To measure the percent constriction due to light, the pupil area was measured after 30 seconds of light exposure in the opposite eye. To measure maximal pupil constriction, 1 to $2 \mu \mathrm{l}$ of $100 \mathrm{mM}$ carbachol was applied to one eye 5 minutes before measurement (Figure 4D). Individual video frames were captured from the beginning and end of the 20 seconds recorded in the dark, at the end of the 30 seconds of light, and 5 minutes after the application of carbachol. Topical application of $0.5 \%$ proparacaine was used to determine if the baseline constriction was due to sensation of the forceps used to hold the eye open.

\section{Additional material}

Additional file 1: RGC and ipRGC birthdating. (A,B) Series of

representative images for birthdating of $\beta$-galactosidase-positive ipRGCs (A) and Brn3a-positive RGCs (B) at PO. Yellow arrowheads denote EdUpositive ipRGCs or Brn3a-positive RGCs, and white arrows denote EdUnegative cells. (C,D) Raw cell counts and proportions of EdU-positive ipRGCs (C) and Brn3a-positive RGCs (D). Scale bars: $50 \mu \mathrm{m}$.

\section{Abbreviations}

CTB: cholera toxin B subunit; dLGN: dorsal lateral geniculate nucleus; E: embryonic day; EdU: 5-ethynyl-2'-deoxyuridine; ipRGC: intrinsically photosensitive retinal ganglion cell; LGN: lateral geniculate nucleus; OPN: olivary pretectal nucleus; P: postnatal day; PLR: pupillary light response; RGC: retinal ganglion cell; SCN: suprachiasmatic nucleus. 


\section{Acknowledgements}

Aaron Stephan and Drs Andy Huberman, Alex Bortvin, Marnie Halpern, Rejji Kuruvilla and Haiqing Zhao for suggestions and proofreading the manuscript, Tara LeGates for help with statistics, and the Johns Hopkins University Mouse Tri-Lab for support. This research was funded by NIH GM076430, NIH EY012716, the David and Lucille Packard Foundation, and the Alfred P Sloan Foundation.

\section{Author details}

'Department of Biology, Johns Hopkins University, Baltimore, MD 21218, USA. ${ }^{2}$ Department of Anatomy and Neurobiology, Virginia Commonwealth University, Richmond, VA 23298, USA. ${ }^{3}$ Retinal Circuit Development and Genetics Unit, N-NRL/NEI/NIH, Bethesda, MD 20892-0610, USA.

\section{Authors' contributions}

DSM and CJS wrote the manuscript. CJS provided data for Figure 1A-D. DSM and JLE provided data for Figure $1 E, 2 B$, and $4 B$. DSM provided data for Figure $1 F, G, 2 C$, and $4 C-F$. TCB provided data for Figure $2 E, F$. DM and WG provided data for Figure 2A,D, and $4 A$. TCB, WG, and SH edited the manuscript.

\section{Competing interests}

The authors declare that they have no competing interests.

Received: 2 November 2010 Accepted: 18 March 2011

Published: 18 March 2011

\section{References}

1. Hatori M, Le H, Vollmers C, Keding SR, Tanaka N, Schmedt C, Jegla T, Panda S: Inducible ablation of melanopsin-expressing retinal ganglion cells reveals their central role in non-image forming visual responses. PLOS ONE 2008, 3:e2451.

2. Goz D, Studholme K, Lappi DA, Rollag MD, Provencio I, Morin LP: Targeted destruction of photosensitive retinal ganglion cells with a saporin conjugate alters the effects of light on mouse circadian rhythms. PLoS ONE 2008, 3:e3153.

3. Altimus CM, Guler AD, Villa KL, McNeill DS, Legates TA, Hattar S: Rodscones and melanopsin detect light and dark to modulate sleep independent of image formation. Proc Natl Acad Sci USA 2008 105:19998-20003.

4. Guler AD, Ecker JL, Lall GS, Haq S, Altimus CM, Liao HW, Barnard AR, Cahill H, Badea TC, Zhao H, Hankins MW, Berson DM, Lucas RJ, Yau KW, Hattar S: Melanopsin cells are the principal conduits for rod-cone input to non-image-forming vision. Nature 2008, 453:102-105.

5. Hattar S, Liao HW, Takao M, Berson DM, Yau KW: Melanopsin-containing retinal ganglion cells: architecture, projections, and intrinsic photosensitivity. Science 2002, 295:1065-1070.

6. Hattar S, Kumar M, Park A, Tong P, Tung J, Yau KW, Berson DM: Centra projections of melanopsin-expressing retinal ganglion cells in the mouse. J Comp Neurol 2006, 497:326-349.

7. Ecker UL, Dumitrescu ON, Wong KY, Alam NM, Chen SK, LeGates T, Renna JM, Prusky GT, Berson DM, Hattar S: Melanopsin-expressing retinal ganglion-cell photoreceptors: cellular diversity and role in pattern vision. Neuron 2010, 67:49-60

8. Quina LA, Pak W, Lanier J, Banwait P, Gratwick K, Liu Y, Velasquez T, O'Leary DD, Goulding M, Turner EE: Brn3a-expressing retinal ganglion cells project specifically to thalamocortical and collicular visual pathways. J Neurosci 2005, 25:11595-11604.

9. Baver SB, Pickard GE, Sollars PJ, Pickard GE: Two types of melanopsin retinal ganglion cell differentially innervate the hypothalamic suprachiasmatic nucleus and the olivary pretectal nucleus. Eur J Neurosci 2008, 27:1763-1770.

10. Young RW: Cell death during differentiation of the retina in the mouse. Comp Neurol 1984, 229:362-373.

11. Farah MH, Easter SS Jr: Cell birth and death in the mouse retinal ganglion cell layer. J Comp Neurol 2005, 489:120-134.

12. Rachel RA, Dolen G, Hayes NL, Lu A, Erskine L, Nowakowski RS, Mason CA: Spatiotemporal features of early neuronogenesis differ in wild-type and albino mouse retina. J Neurosci 2002, 22:4249-4263.
13. Lobe CG, Koop KE, Kreppner W, Lomeli H, Gertsenstein M, Nagy A: Z/AP, a double reporter for cre-mediated recombination. Dev Biol 1999, 208:281-292.

14. Fahrenkrug J, Nielsen $\mathrm{HS}$, Hannibal J: Expression of melanopsin during development of the rat retina. Neuroreport 2004, 15:781-784.

15. Mason CA, Sparrow N, Lincoln DW: Structural features of the retinohypothalamic projection in the rat during normal development. Brain Res 1977, 132:141-148.

16. Speh JC, Moore RY: Retinohypothalamic tract development in the hamster and rat. Brain Res Dev Brain Res 1993, 76:171-181.

17. Sekaran S, Lupi D, Jones SL, Sheely CJ, Hattar S, Yau KW, Lucas RJ, Foster RG, Hankins MW: Melanopsin-dependent photoreception provides earliest light detection in the mammalian retina. Curr Biol 2005, 15:1099-1107.

18. Badea TC, Cahill H, Ecker J, Hattar S, Nathans J: Distinct roles of transcription factors brn3a and brn3b in controlling the development, morphology, and function of retinal ganglion cells. Neuron 2009, 61:852-864.

19. Young MJ, Lund RD: The anatomical substrates subserving the pupillary light reflex in rats: origin of the consensual pupillary response. Neuroscience 1994, 62:481-496.

20. Radel JD, Das S, Lund RD: Development of light-activated pupilloconstriction in rats as mediated by normal and transplanted retinae. Eur J Neurosci 1992, 4:603-615

21. Drager UC: Birth dates of retinal ganglion cells giving rise to the crossed and uncrossed optic projections in the mouse. Proc R Soc Lond B Biol SCi 1985, 224:57-77.

22. Brown NL, Kanekar S, Vetter ML, Tucker PK, Gemza DL, Glaser T: Math5 encodes a murine basic helix-loop-helix transcription factor expressed during early stages of retinal neurogenesis. Development 1998, 125:4821-4833.

23. Wee R, Castrucci AM, Provencio I, Gan L, Van Gelder RN: Loss of photic entrainment and altered free-running circadian rhythms in math5-/mice. J Neurosci 2002, 22:10427-10433.

24. Godement P, Salaun J, Imbert M: Prenatal and postnatal development of retinogeniculate and retinocollicular projections in the mouse. J Comp Neurol 1984, 230:552-575

25. Edwards MA, Schneider GE, Caviness VS Jr: Development of the crossed retinocollicular projection in the mouse. J Comp Neurol 1986, 248:410-421.

26. Gooley JJ, Lu J, Chou TC, Scammell TE, Saper CB: Melanopsin in cells of origin of the retinohypothalamic tract. Nat Neurosci 2001, 4:1165.

27. Shimomura H, Moriya T, Sudo M, Wakamatsu H, Akiyama M, Miyake $Y$, Shibata S: Differential daily expression of Per1 and Per2 mRNA in the suprachiasmatic nucleus of fetal and early postnatal mice. Eur I Neurosci 2001, 13:687-693.

28. Shibata $S$, Moore RY: Development of neuronal activity in the rat suprachiasmatic nucleus. Brain Res 1987, 431:311-315.

29. Pickard GE: Bifurcating axons of retinal ganglion cells terminate in the hypothalamic suprachiasmatic nucleus and the intergeniculate leaflet of the thalamus. Neurosci Lett 1985, 55:211-217.

30. Millhouse OE: Optic chiasm collaterals afferent to the suprachiasmatic nucleus. Brain Res 1977, 137:351-355.

31. Prichard JR, Stoffel RT, Quimby DL, Obermeyer WH, Benca RM, Behan M: Fos immunoreactivity in rat subcortical visual shell in response to illuminance changes. Neuroscience 2002, 114:781-793.

32. Okoyama S, Moriizumi T: Onset of calbindin-D 28K and parvalbumin expression in the lateral geniculate complex and olivary pretectal nucleus during postnatal development of the rat. Int J Dev Neurosci 2001, 19:655-661.

33. Tu DC, Zhang D, Demas J, Slutsky EB, Provencio I, Holy TE, Van Gelder RN: Physiologic diversity and development of intrinsically photosensitive retinal ganglion cells. Neuron 2005, 48:987-999.

34. Johnson J, Wu V, Donovan M, Majumdar S, Renteria RC, Porco T, Van Gelder RN, Copenhagen DR: Melanopsin-dependent light avoidance in neonatal mice. Proc Natl Acad Sci USA 2010, 107:17374-17378.

35. Takada Y, Fariss RN, Tanikawa A, Zeng Y, Carper D, Bush R, Sieving PA: A retinal neuronal developmental wave of retinoschisin expression begins in ganglion cells during layer formation. Invest Ophthalmol Vis Sci 2004, 45:3302-3312. 
36. Jaubert-Miazza L, Green E, Lo FS, Bui K, Mills J, Guido W: Structural and functional composition of the developing retinogeniculate pathway in the mouse. Vis Neurosci 2005, 22:661-676.

37. Lucas RJ, Hattar S, Takao M, Berson DM, Foster RG, Yau KW: Diminished pupillary light reflex at high irradiances in melanopsin-knockout mice. Science 2003, 299:245-247.

doi:10.1186/1749-8104-6-8

Cite this article as: McNeill et al:: Development of melanopsin-based

irradiance detecting circuitry. Neural Development 2011 6:8.

Submit your next manuscript to BioMed Central and take full advantage of:

- Convenient online submission

- Thorough peer review

- No space constraints or color figure charges

- Immediate publication on acceptance

- Inclusion in PubMed, CAS, Scopus and Google Scholar

- Research which is freely available for redistribution

Submit your manuscript at www.biomedcentral.com/submit 